\title{
Magnons coherent transmission and its heat transport at ultrathin insulating ferromagnetic nanojunctions
}

\author{
B. Bourahla ${ }^{\text {a } 1,2}$, A. Khater ${ }^{2}$ and M. Abou Ghantous ${ }^{3}$ \\ ${ }^{1}$ Laboratoire de Physique de l'Etat Condensé, université du Maine, 72085 Le Mans, France \\ ${ }^{2}$ Laboratoire de Physique et Chimie Quantique, université M. Mammeri de Tizi Ouzou, Algérie \\ ${ }^{3}$ Department of Physics, Texas A and M University at Qatar, Education City, PO Box 23874, Doha, \\ Qatar
}

\begin{abstract}
A model calculation is presented for the magnons coherent transmission and corresponding heat transport at magnetic insulating nanojunctions. The system consists of a ferromagnetically ordered ultrathin insulating junction between two semi-infinite ferromagnetically ordered leads. Spin dynamics are analyzed using the equations of motion for the spin precession displacements, valid for the range of temperatures of interest. Coherent scattering cross-sections at the junction boundary are calculated using the phase field matching theory, for all the incidence angles on the boundary from the lead bands, for arbitrary angles of incidence, at variable temperatures, and for different nano thicknesses of the ultrathin junction. The model is general; it is applied in particular to the $\mathrm{Fe} / \mathrm{Gd} / \mathrm{Fe}$ system with a sandwiched ferromagnetic $\mathrm{Gd}$ junction. It yields also the thermal conductivity due to the magnons coherent transmission between the two leads when these are maintained at slightly different temperatures. The calculation is carried out for state of the art values of the exchange constants, and elucidates the relation between the coherent scattering transmission of magnons and their thermal conductivity, for different thicknesses.
\end{abstract}

\section{Introduction}

Nanomaterial systems may be considered as solids for which a parameter at least varies on a scale of few nanometers up to the submicroscopic. Due to their material confinement, these nanostructures present a significant atomic proportion of their material at their surfaces. They consequently have structural [1], magnetic [2], and electronic [3] properties which are quite different from those of the bulk materials.

The study of the localization and the coherent scattering of excitations in low dimensional systems at embedded nanostructures, has been of intensive interest in the last decade, in view of the potential use of these systems for nanotechnology. The majority of this research work has been directed to the study of the electronic scattering and quantum transmission in low dimensional systems. The comprehension of electronic transport in these systems and its generalization to multi-

\footnotetext{
a e-mail : bourahla_boualem@yahoo.fr
} 
channel systems are provided by the models of Landauer [4], and Büttiker [5], which established a relation between the conductance of a given nanojunction and its scattering matrix.

In this communication, we present a model to analyze quantitatively in a first approach the coherent magnons transport via an insulating magnetic nanojonction, which may be prepared as an ultrathin film of gadolinium Gd inserted between two semi-infinite fcc leads of iron Fe. The Fe/ $\mathrm{Fe} / \mathrm{Gd}_{\mathrm{n}} / \mathrm{Fe}$ system is presented schematically in Fig.1. The thickness of the ultrathin Gd film shall be treated as a variable, ranging from one to four atomic layers. In particular we show that this nanojonction is capable of a selective transmission of magnons as a function of its thickness, which is due to the coupling between the incident propagating magnon modes of the semi-infinite Fe leads located on both sides of the nanojonction and the localized modes characteristic of the local spin dynamics at the nanojonction.

In the following section 2 we present the model spin dynamics for this system (2.1), and the heat transport properties of these junctions (2.2), respectively.

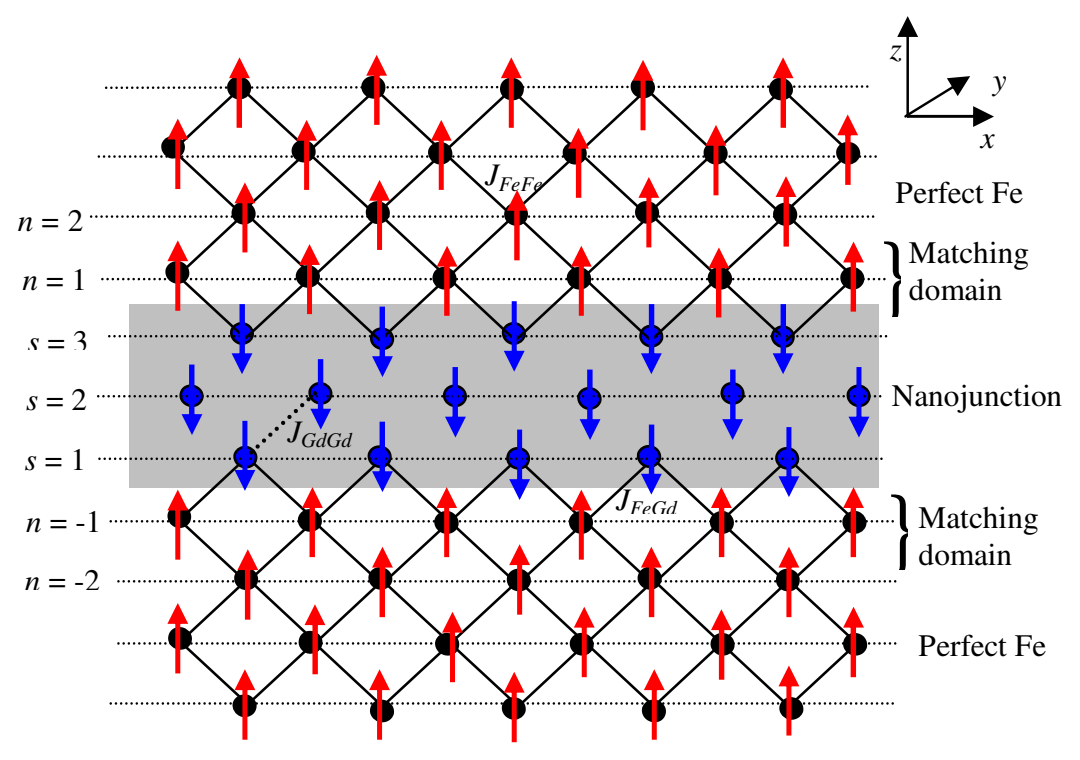

Fig.1. Schematic representation of an ultrathin film of Gd sandwiched between two fcc semi-infinite leads of $\mathrm{Fe}$. The gray area constitutes the insured Gd film.

\section{Nanojunction spin dynamics}

\subsection{Magnons transmission and reflection}

The ground state of the system is described by the Heisenberg Hamiltonian:

$\mathrm{H}=-\sum_{p \neq p}, J_{p p}, \boldsymbol{S}_{p} \cdot \boldsymbol{S}_{p}$,

where $\boldsymbol{S}_{p}\left(\boldsymbol{S}_{p}\right)$ are the spin vectors, and $p \equiv\left[p_{x}, p_{y}, n(s)\right]$, here the indices $p_{x}, p_{y}$, and $n(s)$ describe the sites along the $\mathrm{x}, \mathrm{y}$, and $\mathrm{z}$ directions, respectively. The exchange interactions between nearest neighbors $J_{p p^{\prime}}=J$ are considered for adjacent sites $p\left(p^{\prime}\right)$ of the system. The exchange constant for ferromagnetic coupling between nearest neighbor sites is $J>0$.

The equation of motion for a spin vector $S_{p}$ located at an Fe site in the leads to the left and right of the nanojunction, as in Fig.1, may be given in the linear approximation, [6], for the range of temperatures of interest $[300,800] \mathrm{K}$, since the critical order disorder temperature for iron is much higher than that for gadolinium. For sites $p$ and $p$ ' which are distant from the Gd nanojunction to the 
left and right of the interval, $n \in[-1,1]$ of Fig.1, the equations of motion may be cast, in the matrix form:

$$
\left[\Omega I-D\left(J, z, \mathrm{e}^{j \varphi x}, \mathrm{e}^{j \varphi y}\right)\right] \mid \zeta \pm_{p}>=0
$$

$\zeta \pm_{p}>$ corresponds to the vector describing the spin precessions. $\Omega=\omega / \omega_{0}=\hbar \omega / J S$ is the normalized dimensionless frequency, and $I$ is the unit matrix. $D\left(J, z \mathrm{e}^{j^{*} \varphi x}{ }_{. .} \mathrm{e}^{* \varphi y}\right)$ is the dynamic matrix of the perfectly ordered magnetic Fe lead system. Given the symmetry of the system we define the quantities $\varphi_{x}=k_{x} a, \varphi_{y}=k_{y} a$, and a generalized $\eta$, to depict the Bloch phase factors along the $\mathrm{x}, \mathrm{y}$ and $\mathrm{z}$-directions, respectively. The propagating and evanescent modes are described by the doublet phase factors $\left\{z, z^{-1}\right\}$.

The propagating magnon modes are determined from the condition that $|\eta|=1$, and the evanescent modes from the condition $|\eta|<1$, [7]. The exact solutions for each doublet are obtained as a function of the frequencies $\Omega$ by linearizing the initial system of equations [8].

For a magnon mode $\eta_{l}$ incident at a frequency $\Omega$ along the $\mathrm{z}$ direction as in Fig.1, the spin wave scattering due to the nanojunction are coherent reflected and transmitted fields. Let $r_{i j}$ and $t_{i j}$ denote the reflection and transmission coefficients that describe the scattering. For sites in the perfect lead waveguides for which $n \leq-2$, and $n \geq 2$, the spin fluctuations field $\mid \zeta \pm_{p}>$ may be expressed in terms of an appropriate superposition of the incident and scattered eigenmodes of the perfect lead waveguide at the same frequency.

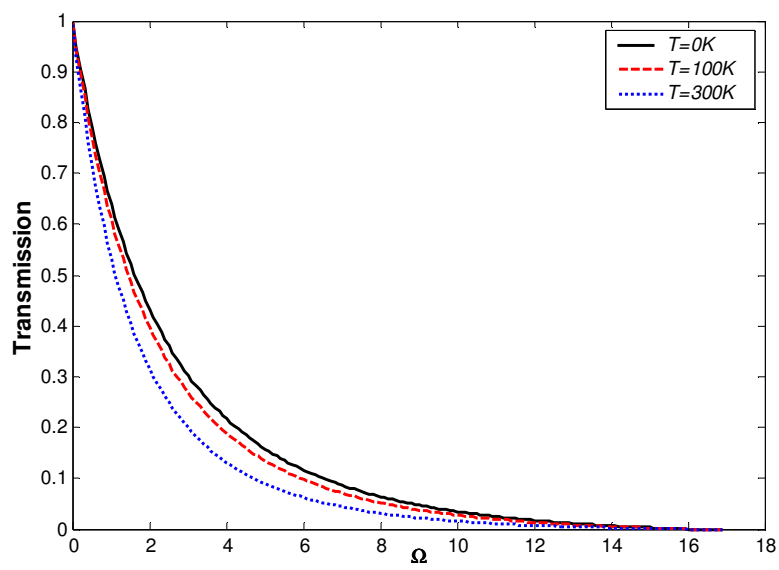

Fig.2. Magnon transmission coefficients for the nanojunction system $\mathrm{Fe} / \mathrm{Gd}_{1} / \mathrm{Fe}$ with a single $\mathrm{Gd}$ atomic plane, for temperatures $T=0 \mathrm{~K}, 100 \mathrm{~K}$, and $300 \mathrm{~K}$.

Consider a Hilbert space constructed from the basis vectors $[|\mathrm{R}>,| \mathrm{T}\rangle]$ for the reflection and transmission into the different eigenmodes, and let $\mid \zeta \pm_{p}($ nano $)>$ group the spin precession displacements for an irreducible set of spins in the nanojunction domain, for which $s=1,2,3$ along the $\mathrm{z}$ axis. The equations of motion for this domain, coupled to the rest of the system may be written consequently in terms of the vector $\left[\mid \zeta \pm_{p}(\right.$ nano $\left.)>,|R>| T>,\right]$. The transformations connecting the spin fluctuations field yield then a square linear inhomogeneous system of equations of the form

$$
\left.\left[\Omega I-D\left(J, J_{G d}, J_{G d F e}, z, \mathrm{e}^{j^{*} \varphi x},, \mathrm{e}^{j^{*} \varphi y}\right)\right]\left[\zeta \pm_{p}(\text { nano })>,|\mathrm{R}>,| \mathrm{T}>\right]=-\mid \mathrm{IH}, \eta_{l}, \varphi_{y}>\right] .
$$

The vector $\left.-\left|\mathrm{IH}, \eta_{l}, \varphi_{y}\right\rangle\right]$, mapped appropriately onto the basis vectors in the constructed Hilbert space, regroups the inhomogeneous terms describing the incoming magnons.

In the scattering phenomena, the reflection and transmission effects are described in terms of the scattering matrix elements which are given explicitly by the reflection coefficient $R_{i j}$ for the backward scattered or reflected waves $j$, and by the transmission coefficient $T_{i j}$ for the forward scattered or transmitted wave $j$, per incident propagating mode $i$. 


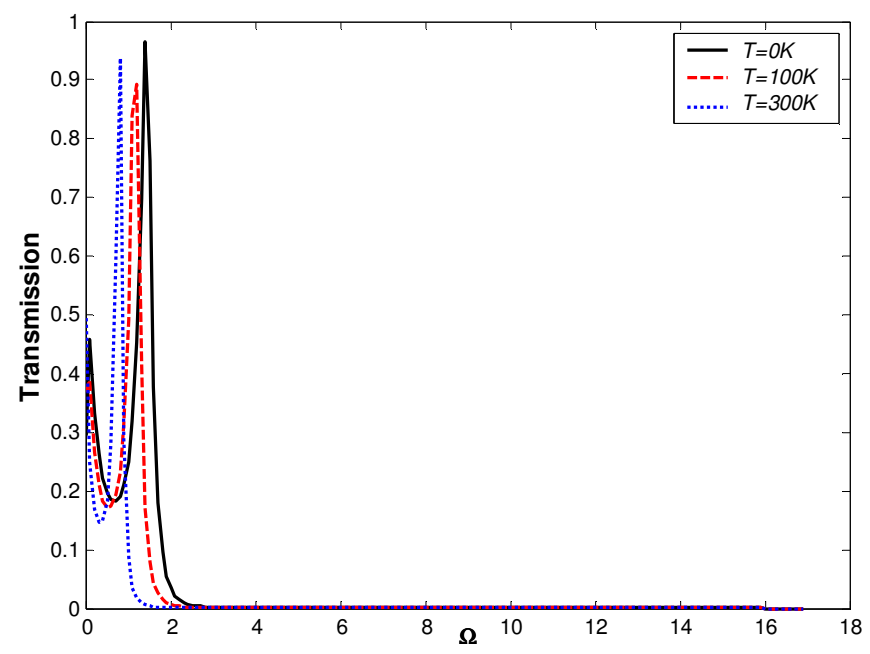

Fig.2. As in Fig.2 for the nanojunction system $\mathrm{Fe} / \mathrm{Gd}_{3} / \mathrm{Fe}$ with a single $\mathrm{Gd}$ atomic plane

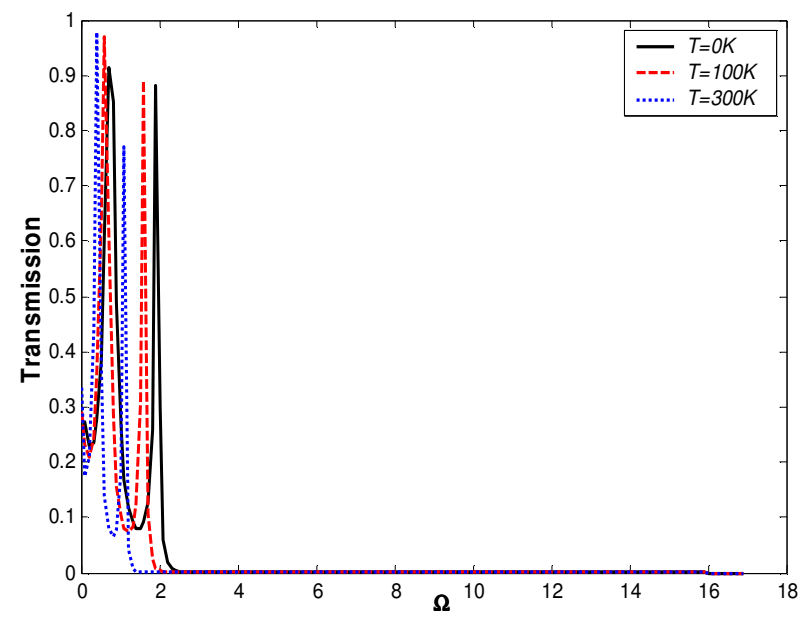

Fig.4. As in Fig.2 for the nanojunction system $\mathrm{Fe} / \mathrm{Gd}_{4} / \mathrm{Fe}$ with a single $\mathrm{Gd}$ atomic plane

The coherent scattering of the magnons is described, as for other excitations, in terms of the Landauer-Büttiker like scattering matrices. For different incident magnons $i$, the solutions of Eq.(3) yield the reflection and transmission coefficients $R_{i j}$ and $T_{i j}$, and the spin fluctuations vector $\zeta \pm_{p}($ nano $)>$ for the irreducible set of spins of the nanojunction domain. The reflection and transmission scattering cross sections $r_{i j}$ and $t_{i j}$ are then given at the scattering frequency $\Omega$, as

$$
\begin{aligned}
& r_{i j}=\left(v_{g j} / v_{g i}\right)\left|R_{i j}\right|^{2} \\
& t_{i j}=\left(v_{g j} / v_{g i}\right)\left|T_{i j}\right|^{2}
\end{aligned}
$$

To ensure the unitarity of the scattering matrix, the scattering cross sections are normalized with respect to the group velocities of the magnons. In this respect $v_{g i}$ is the group velocity of the eigenmode $i$, it is equal to zero for evanescent modes.

We can finally define the total reflection and transmission cross sections for a given eigenmode $i$ at frequency $\Omega$, by summing over all the contributions of the scattered magnons, to yield $r_{i}\left(\Omega, \varphi_{y}\right)=\sum_{j} r_{i j}\left(\Omega, \varphi_{y}\right)$ $t_{i}\left(\Omega, \varphi_{y}\right)=\sum_{j} t_{i j}\left(\Omega, \varphi_{y}\right)$. 
Furthermore, it is useful to define the total magnon nanojunction conductance as

$\sigma\left(\Omega, \varphi_{y}\right)=\sum_{i} \sum_{j} t_{i j}\left(\Omega, \varphi_{y}\right)$.

This describes effectively the overall transmission of an ultrathin insulating magnetic nanojunction at a given frequency $\Omega$, for a given incidence angle.

\subsection{Heat transport across the nanojunction}

We suppose that the two semi-infinite Fe systems located on both sides of the nanojonction are held respectively at the temperatures $T_{L}$ and $T_{R}$. The thermal transport due to magnon transmission at the nanojunction is associated with the small temperature difference $\left(T_{L}-T_{R}\right)>0$ across the nanojonctions. The magnons which are incident from the left hand reservoir towards the right hand reservoir, and vice verse, contribute to the thermal exchange between the two reservoirs.

The net thermal transport from the left to the right, across the nanojunction may be derived for a small temperature difference $\Delta T$ in the form

$$
\kappa=\frac{\dot{Q}}{\Delta T}=\frac{1}{d} \sum_{j} \frac{1}{(2 \pi)^{3}} \int d^{2} q v_{g j}(q) \hbar \omega_{j}(q) t_{j}\left(\omega_{j}, q\right) \frac{\partial n\left(T_{L}, \omega_{j}(q)\right)}{\partial T} .
$$

$\kappa$ is the thermal transport associated with the coherent magnon transmission, $q$ the magnon wavevector, $\omega_{j}(q)$ the dispersion relation of $j^{\text {th }}$ magnon branch in the semi-infinite lead system, and $v_{g j}(q)$ depicts the magnon group velocities corresponding to the propagation of a magnon across the junction. In particular $t_{j}\left(\omega_{j}, q\right)$ is the transmission coefficient across the nanojunction domain for magnons at frequencies $\omega_{j}$, and wave vector $q$. At a given absolute temperature $T$ of the system, $n\left(T_{L}, \omega_{j}(q)\right)$ is related to Bose-Einstein distribution of magnons.

\section{Results and discussion}

The model calculations are next applied numerically in this section for the $\mathrm{Fe} / \mathrm{Gd}_{\mathrm{n}} / \mathrm{Fe}$ nanojunctions, for three different thicknesses $n=1,3$, and $4 \mathrm{Gd}$ atomic planes. To carry out the numerical applications of the theoretical model, we need values for the system exchange constants.

In a recent work [9] we have used a set of values from previous work [10], which uses the effective field theory over an Ising Hamiltonian to calculate the thermal averages of the magnetizations on the atomic spin layers at a nanojunction interface. The numerical results correspond hence to a relatively weak $J_{F e G d}$. Contrary to this previous work, we take into consideration, in the present work, the possibility of quite different exchange couplings between the $\mathrm{Fe}$ and $\mathrm{Gd}$ atoms at the interfaces of the nanojunction [11], and in certain GdFe compounds [12-13]. These values for the $J_{F e G d}$ magnetic exchange, of which we were not aware, have been reported previously in the literature. In the present work the values of the exchange interactions between interface sites for the sandwiched $\mathrm{Fe} / \mathrm{Gd} / \mathrm{Fe}$ nanojunction system are given as follows: $J_{\mathrm{FeFe}}=130 \times$ $10^{-23} \mathrm{~J}=8.1 \mathrm{meV}, J_{G d G d}=35 \times 10^{-23} \mathrm{~J}=2.1 \mathrm{meV}$, and $J_{F e G d}=-98 \times 10^{-23} \mathrm{~J}=-6.2 \mathrm{meV}$. The spin are considered $S_{F e}=1, S_{G d}=7 / 2$.

The numerical results, which consequent to these new values, for the magnon coherent transmission via the magnetic nanojonction $\mathrm{Fe} / \mathrm{Gd} / \mathrm{Fe}$ are presented in Figs.2, 3 and 4, in each case for three different temperatures, $T=0 \mathrm{~K}, 100 \mathrm{~K}$ and $300 \mathrm{~K}$, and normal incidence $\varphi_{x}=\varphi_{y}=0$. These results are effectively for different material nanojunctions corresponding to different thicknesses of one, three and four atomic Gd planes. Note that the limits of the transmission spectra correspond to the cut-off frequencies of the corresponding dispersion curves in the Fe leads. In Fig. 3 the spectral Fabry-Perot peak at $\Omega=1.50$ is observed for $T=0 \mathrm{~K}$ which signals the important transmission at this frequency. The number of Fabry-Perot peaks increase and appear at different frequencies for nanojunctions of greater thickness as in Fig.4. As may be observed these peaks shift systematically to lower frequencies for higher temperatures. 
As for the heat transport due to the coherent transmission of electrons and phonons, the corresponding heat transport due to magnons can be calculated for the present case using Eq.9. There is indeed some experimental interest in this since several groups have recently used heat transport to probe spin excitations in quantum magnets [14]. We have also calculated the thermal conductivities for our present nanojunction systems with the strong $J_{F e G d}$ exchange in the range of $100 \mathrm{~K}$ to $700 \mathrm{~K}$. For example, for the studied case, the 3-atomic planes nanojunction shows a more important thermal conductivity in the range of $100 \mathrm{~K}$ to $500 \mathrm{~K}$. This is primarily due to the Fabry-Perot peak at relatively small frequencies. The decrease of the thermal conductivity $\kappa$ for this nanojunction with increasing temperature comes partly from the decreasing transmission cross section at higher frequencies, but principally due to the decreasing magnetization with temperature of the central $s=2$ atomic plane in the Gd junction, which is less influenced than the $s=1$ and $s=3$ planes by the magnetic ordering effects of the adjacent Fe atomic planes at $n= \pm 1$.

These results for the heat transport are however quite similar to our recent results [9] with the weaker $J_{F e G d}$ exchange, and need not be presented here. This leads to the conclusion that whereas the transmission spectra are strongly modified by the $J_{F e G d}$ exchange, the heat transport is not.

In conclusion, we present a model calculation for the study the magnonic conductance and the heat transport of magnons via magnetic nanojunction in fcc nanojunction system $\mathrm{Fe} / \mathrm{Gd} / \mathrm{Fe}$. In particular, we analyze the magnetic properties induced by the insertion magnetic layers of $\mathrm{Gd}$ between the two semi-infinite fcc leads of Fe. We examine three cases corresponding, respectively, to one layer, three and four atomic layers of Gd. The analysis of the transmission spectra and the thermal conductivity of the sandwich system show the central role of the number of Gd layers for the dynamics of the sandwich model. It can be used as model for the study magnetic materials built in the same way as the studied system.

\section{References}

1. S. Rousset et al., Materials Sc. Eng. B 96, 169 (2002)

2. A. Vindigni, A. Rettori, M.G. Pini, C. Carbone, P. Gambardella, Appl. Phys. A 82, 385 (2006)

3. J.N. Crain and D.T. Pierce, Science 7, 703 (2005)

4. R. Landauer, Philos. Mag. 21, 863 (1970)

5. M. Büttiker, Phys. Rev. Lett. 57, 1761 (1986)

6. A. Khater and M. Abou Ghantous, Surf. Sci. Lett. 498, L97 (2002); M. Abou Ghantous and A. Khater, Eur. Phys. J. B 12 (1999)

7. J. Szeftel and A. Khater, J. Phys. C: Solid State Phys. 20, 4725 (1987)

8. C. Berthod, F. Gagel and K. Maschke, Phys. Rev B 50, 18299 (1994)

9. A. Khater, B. Bourahla, M. Abou Ghantous, R. Tigrine and R. Chadli, Eur. Phys. J. B: Cond. Matter 82, 53 (2011)

10. M. Fresneau, G. Le Gal, A. Khater, J. Mag. Mag. Mat 130, 63 (1994); M. Fresneau, A. Virlouvet, and A. Khater, J. Mag. Mag. Mat. 202, 220 (1999); A. Khater, M. Abou Ghantous, M. Fresneau, J. Mag. Mag. Mat. 247, 305 (2002)

11. Y. Yafet, J. Kow, M. Hong, C.F. Majkrzka, and T. Obrien, J. App. Phys. 63, 3453 (1988)

12. M. Loewenhaupt, P. Tils, D.P. Middleton, K.H.J. Buschow, and R. Eccleston, J. Mag. and Mag. Mat. 129, L151 (1994)

13. M. Loewenhaupt, P. Tils, K.H.J. Buschow and R. Eccleston, J. Mag. and Mag. Mat. 152, 10 (1996)

14. S.Y. Li, L. Taillefer, C. H. Wang and X. H. Chen, Phys. Rev. Lett. 95, 156603 (2005) 